\title{
Feature Analysis of Mechanical and Electronic Signals Based on Grey Neural Network Prediction
}

\section{He Yuyang}

School of Electronic Engineering, Xi'an University of Science and Technology, Xi'an, China

Keywords: fault diagnosis; electronic signal; wavelet entropy; support vector machine

\begin{abstract}
The mechanical performance of high-speed train bogies has an important impact on the safety and comfort of the entire operating train system. The main purpose of this paper is to apply the intelligent signal processing methods such as wavelet entropy and support vector machine to the processing of massive online monitoring data of high-speed train bogies, seek the characteristic parameters that are generally applicable to the fault state of high-speed trains, and judge and identify the fault state of the main damping components of the bogies. This paper is aimed at discussing the applicability of wavelet entropy feature extraction method in vibration signal analysis of high-speed trains.
\end{abstract}

\section{Introduction}

With the large-scale acceleration of high-speed railways, more and more attention has also been paid to the safety and comfort of high-speed trains. The real-time monitoring and data acquisition system in the operation of high-speed trains has been continuously developed and improved. The sensors distributed throughout the train collect a large amount of operational data which contains rich information reflecting the fault state and extent of the train. How to effectively analyze and use these data to extract the universally applicable characteristic quantities that can respond to fault state, mine and invert the health state of high-speed train bogies through the massive monitoring data and guide the health maintenance of train is a high-profile hot and difficult issue [1-4] at present.

\section{Fault Diagnosis of High-speed Train}

From the basic theory of vehicle-track coupling dynamics, it is known that the vibration of various parts of the bogie of a high-speed train in operation is caused by the uneven excitation of the track. While the train works in the normal conditions, the vibration (displacement and acceleration) of the bogie at each position shows a strong regularity, and such regularity is inextricably linked with the parameters of the train itself. When the key components of the bogie have different degrees of failure and fault, the normal vibration law will disappear, and the signal will show the unique vibration law in response to the fault state. The vibration law will differ for different fault locations and different failure extents.

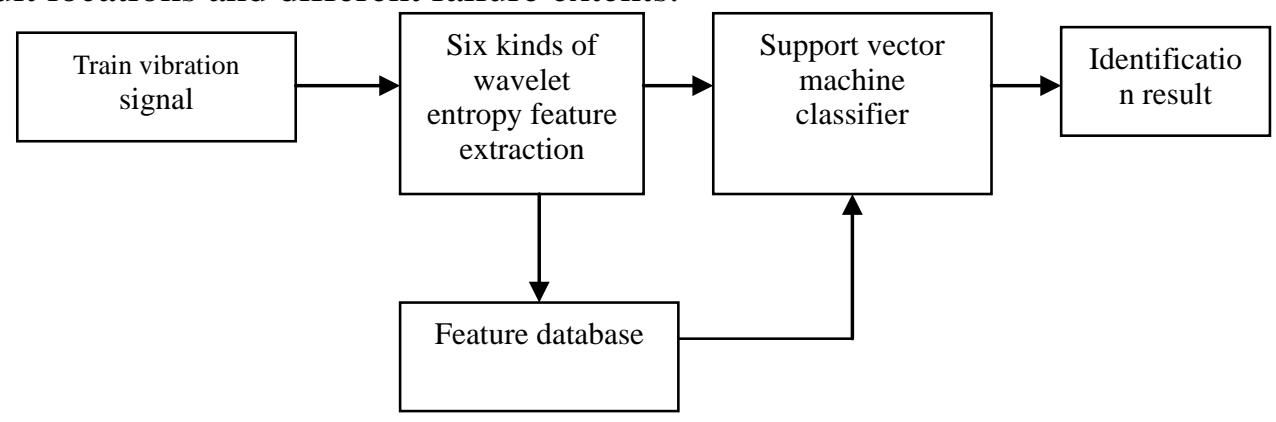

Fig. 1 Flow Block Diagram of Vibration Signal Processing

Fig. 1 is a flow block diagram of the vibration signal processing. The normal state on the bogie 
and the typical fault modes of the three key vibration damping components (transverse damper fault, anti-snakelike damper fault and air loss of air spring) of the high-speed train are selected for comparison test, and the vibration signals collected in the test from various parts of the high-speed train bogie are analyzed. The wavelet packet decomposition threshold denoising method is used to preprocess the vibration signals collected from various parts of the bogie to eliminate noise interference. Then the denoised signal is decomposed by wavelet, and five typical wavelet entropy features are extracted on the multiscale reconstruction coefficients of wavelet decomposition. Finally, the extracted feature vectors are trained and identified by the support vector machine classifier to obtain the fault diagnosis result of the bogie and the identification result is judged and analyzed. The experimental results show that this method overcomes the shortcomings of the traditional signal processing methods, effectively extracts the vibration fault features of the key components of the bogie, and accurately realizes the classification of the four typical working states (bogie normality, transverse damper fault, anti-snakelike damper fault and air loss of air spring), and is an effective method for feature analysis on mechanical fault of the bogies.

\section{Wavelet Entropy Feature of the Signal}

Wavelet entropy theory is a time-frequency analysis method first proposed by Blanco et al. in 1998 based on the basic definition of wavelet transform theory. Proposed on the basis of the concept of physical entropy, the information entropy describes the randomness and uncertainty of the system. Wavelet entropy combines the advantages of these two methods in signal processing and is suitable for extracting the statistical property of the complexity of irregular nonlinear signals. The vibration of the train system is related to the driving speed and the state of the passing route, which is a complex coupling relationship. When the damping component of the bogie has different degrees of fault, the fault state vibration signals collected by the sensors throughout the bogie are the typical non-stationary random signals containing various complex frequency components. The wavelet transform can be used for multi-scale decomposition of the vibration signal and the wavelet entropy features reflecting the complexity and disorder degree of the signal are extracted in each frequency range.

According to the characteristics of the vibration signal, five kinds of wavelet entropy are selected for feature extraction and analysis on the fault data of the high-speed train bogie. The calculation methods of these five wavelet entropies are briefly described below.

\subsection{Wavelet Energy Entropy, WEE}

After $m$ layers of wavelet decomposition is performed to signal ${ }^{x(t)}$, the wavelet energy spectrum $E=E_{1}, E_{2}, \cdots, E_{m}$ of each frequency band is obtained according to the reconstruction coefficient of wavelet decomposition reconstruction at each scale.

Given $p_{j}=E_{j} / E$ and $\sum_{j} p_{j}=1$, the calculation formula of wavelet energy entropy is:

$$
W_{E E}=-\sum_{j} p_{j} \log p_{j}
$$

\subsection{Wavelet Time Entropy, WTE}

Divide the signal into $\mathrm{L}$ mutually disjoint intervals $\left\{Z_{l}=\left[s_{l-1}, s_{l}\right], l=1,2, \cdots L\right\}, p^{m}\left(Z_{l}\right)$ is the probability value of the wavelet decomposition coefficient $d_{j}(k) \in W(m ; w, \delta)$ falling in the interval $^{Z_{l}}$. At the wavelet decomposition scale $\mathrm{j}$, the following definition of the wavelet time entropy can be obtained:

$$
W_{T E j}(m)=-\sum p^{m}\left(Z_{l}\right) \log \left(p^{m}\left(Z_{l}\right)\right), m=1,2, \cdots M
$$




\subsection{Wavelet Singular Entropy, WSE}

The calculation formula of the wavelet singular entropy is:

$$
W S E_{k}=\sum_{i=1}^{k} \Delta p_{i}
$$

In the above formula, $\Delta p_{i}=-\left(\lambda_{i} / \sum_{j=1}^{l} \lambda_{j}\right) \log \left(\lambda_{i} / \sum_{j=1}^{l} \lambda_{j}\right)$ is the expression of the incremental wavelet singular entropy in $j$ th order. Wavelet singular entropy is used to describe the frequency components of the analyzed signal and the distribution characteristics of the frequency components.

\subsection{Wavelet Time-Frequency Entropy, WTFE}

The wavelet time-frequency entropy is based on the discrete wavelet decomposition $W T_{x}\left(a=2^{j}, t=k T\right)$ of the signal. The calculation formula is:

$$
\begin{aligned}
& \qquad W T F E_{x}(k T, j)=\left[E_{W T_{x}}(t=k T), E_{W T_{x}}\left(a=2^{j}\right)\right] \\
& E_{W T_{x}}\left(a=2^{j}\right)=-\sum_{k} P_{W T_{k}\left(a=2^{j}, t=k T\right)}(t=k T)=-\sum_{j} P_{W T_{j}\left(a=2^{j}, t=k T\right)} \ln P_{W T_{k}\left(a=2^{j}, t=k T\right)}
\end{aligned}
$$

\subsection{Wavelet Average Entropy, WAE}

The time-frequency plane formed by the coefficients after wavelet transform of the signal is divided into $\mathrm{N}$ time-frequency regions, and the energy in each region is $E_{i}(i=1,2, \ldots . N)$, and the total energy is $E=\sum_{i=1}^{N} E_{i}$. Define $P_{i}=E_{i} / E$, then $\sum_{i=1}^{N} P_{i}=1$. Hence the obtained calculation formula of wavelet average entropy is:

$$
W \mathrm{~A} E=-\sum_{i=1}^{N} P_{i} \ln P_{i}
$$

The above five wavelet entropies define and describe the statistical properties of the complexity of the analyzed signals at various scales of wavelet decomposition from different angles and levels.

\section{Analysis of Examples}

\subsection{Data sources and experimental scheme}

The fault condition of the bogie mainly involves the transverse damper fault, the anti-snakelike damper fault, air loss of the air spring and the original train scheme (fault-free state), and the added rail excitation is the track spectrum of Wuhan-Guangzhou Line. The operating speed in each working condition is increased by $40 \mathrm{~km} / \mathrm{h}, 80 \mathrm{~km} / \mathrm{h}, 120 \mathrm{~km} / \mathrm{h}, 140 \mathrm{~km} / \mathrm{h}, 160 \mathrm{~km} / \mathrm{h}, 200 \mathrm{~km} / \mathrm{h}, \ldots$ until it stops after instability. It runs for about 3.5 minutes at each speed, with a sampling frequency of $243 \mathrm{~Hz}$. Fig. 2 shows the time-domain signal and amplitude spectrum of transverse vibration acceleration at Location 1 of Bogie I under four working conditions, in which (a) indicates the original train (fault-free state), and (b) represents the air spring without air, (c) shows anti-snakelike damper fault, (d) describes transverse damper fault. 

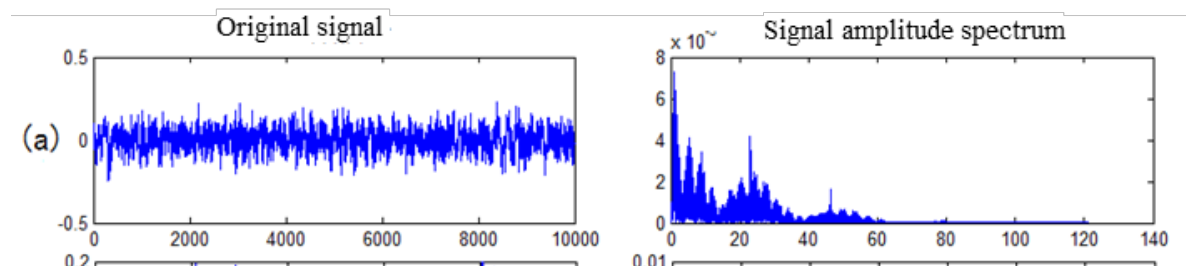

(b)
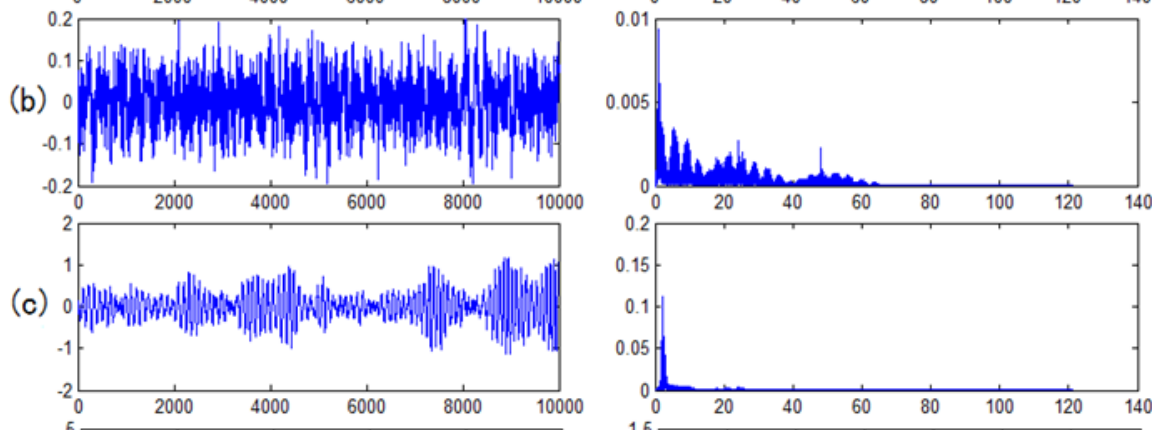

(d)
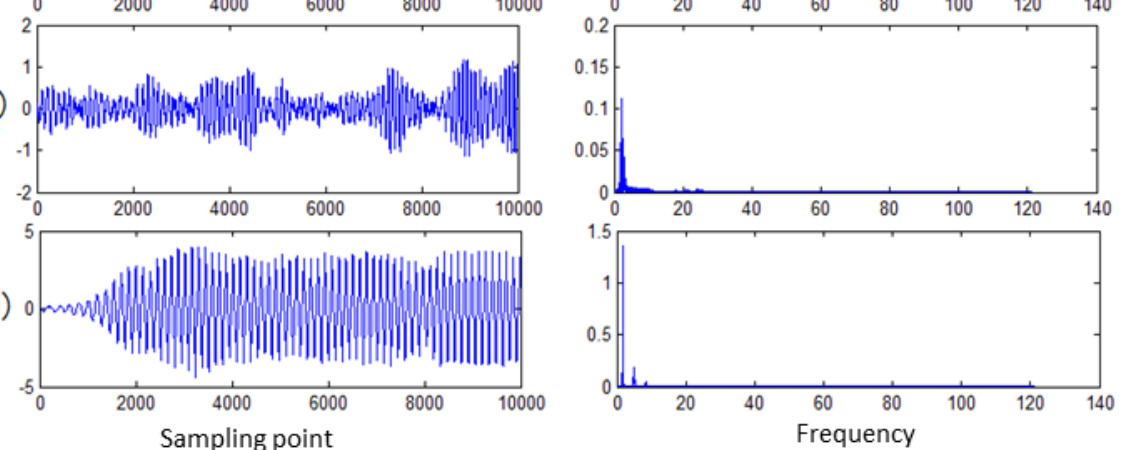

Fig. 2 Time-Domain Signal and Amplitude Spectrum under Four Working Conditions

\subsection{Wavelet entropy feature extraction}

Currently, there is no clear or uniform standard for the selection of wavelet mother functions for wavelet analysis of signals. Considering that the train fault vibration signal contains more complex frequency components, the signal has non-stationary characteristics such as instantaneity, singularity and mutability, the orthogonal wavelet base db4 is selected, and the time-frequency compactness and high regularity make the $\mathrm{db}$ wavelet have better high time-frequency resolution. According to the sampling frequency and the characteristic frequency of the fault signal, the number of wavelet decomposition layers is initially determined to be 4 layers. The data is selected as the longitudinal displacement signal at the anti-snakelike damper of the rear frame of a bullet train at a speed of $200 \mathrm{~km} / \mathrm{h}$, with a sample length of 5 seconds and 1215 sampling points. Fig.3 shows three wavelet entropy features of the bogie under four typical fault conditions.

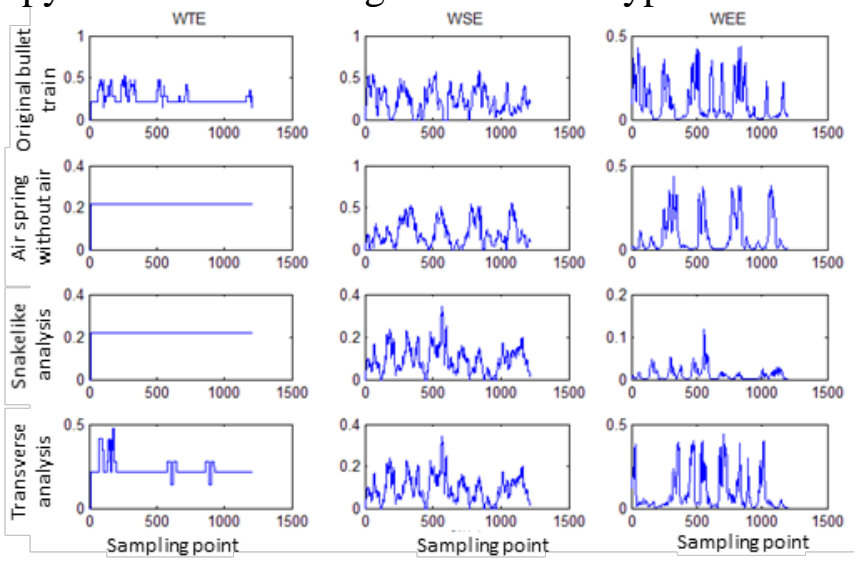

Fig.3 Three Wavelet Entropy Features of Bogie under Fault Conditions

It can be seen from Fig. 3 that the fluctuation range of the eigenvalues obtained for various faults in the single wavelet entropy feature is not the same, indicating that complexity of signal analysis under different working conditions is different from different perspectives. For the fault vibration signal of high-speed train bogie, WEE describes the vibration energy distribution of the bogie fault signal in the time domain and the frequency domain space. WTE has a strong ability in detecting 
the composition and parameter changes of the bogie fault vibration signal.

WSE is a parameter to measure the uncertainty and complexity of the energy distribution of the analyzed signal in time-frequency space. The simpler the signal is, the less and more centralized the mode is included and the smaller the signal uncertainty is, hence the smaller the WSE is. Otherwise, the entropy value will be greater. The WSE value of the original train and the air spring without air is between 0 and 0.5 , while the WSE of the other two conditions is between 0 and 0.2 , which indicates that the vibration signal of the bogie at $200 \mathrm{~km} / \mathrm{h}$ in anti-snakelike transverse damper faults is less complex than the signals in other two conditions at the same speed, and the train is closer to the unstable state in such faults. Table 1 shows the critical speeds in the four typical fault conditions of the bogies obtained from the dynamic test of the rolling test rig, this indicates conclusions obtained from dynamic experiments are consistent with those from the wavelet entropy features analysis of the signal.

Table 1 Critical Speeds in Four Typical Failure Conditions of the Bogies

\begin{tabular}{lc}
\hline Fault type & Critical speed $(\mathrm{km} / \mathrm{h})$ \\
\hline Transverse damper fault & 250 \\
Anti-snakelike damper fault & 220 \\
Air loss of air spring & 380 \\
Original train & Stable at 480 \\
\hline
\end{tabular}

Four fault conditions cannot be distinguished well with a view to single one of the wavelet entropy features, and the working conditions that can be distinguished by various wavelet entropy features is complementary mutually. If multiple wavelet entropies are combined into feature vectors, various faults will be distinguished well in this high-dimensional feature space.

\section{Conclusion}

According to the basic theory of vehicle-track coupling dynamics, the typical characteristics of high-speed train operating in fault are analyzed. Taking the vibration signal of four typical working conditions of main damping components of bogie as the analysis target, the wavelet packet threshold method is used for noise elimination first, and then five kinds of wavelet entropy features of the vibration signal in the fault state are extracted, and the separability of various fault states is analyzed on a space formed by the five-dimensional entropy feature. Finally, the simulation data is analyzed and processed and the support vector machine is selected as the classifier, which gives a satisfactory identification effect. The impact of running speed of the train on identification rate is analyzed briefly. Thereby the validity of wavelet entropy feature for vibration signal analysis of high-speed trains is verified.

\section{References}

[1] Kiratitanavit W, Xia Z, Yu S, Facendola P, Ramanathan R, Mosurkal R, Nagarajan R. Bio-derived phytic acid complex as a char forming additive for polypropylene. InABSTRACTS OF PAPERS OF THE AMERICAN CHEMICAL SOCIETY 2016 Aug 20 (Vol. 252). 115516 TH ST, NW, WASHINGTON, DC 20036 USA: AMER CHEMICAL SOC.

[2] Xia Z, Yu S, Kiratitanavit W, Kumar J, Mosurkal R, Nagarajan R. Bio-based intumescent flame retardant coating based on synergistic combination of phytic acid and tannic acid for nylon-cotton blends. InABSTRACTS OF PAPERS OF THE AMERICAN CHEMICAL SOCIETY 2017 Aug 20 (Vol. 254). 1155 16TH ST, NW, WASHINGTON, DC 20036 USA: AMER CHEMICAL SOC.

[3] Ghebrebrhan M, Aranda F, Walsh G, Ziegler D, Giardini S, Carlson J, Kimball B, Steeves D, Xia Z, Yu S, Kingsley E. Textile Frequency Selective Surface. IEEE Microwave and Wireless Components Letters. 2017 Nov; 27(11):989-91.

[4] Kurup P, Sullivan C, Hannagan R, Yu S, Azimi H, Robertson S, Ryan D, Nagarajan R, 
Ponrathnam T, Howe G. A Review of Technologies for Characterization of Heavy Metal Contaminants. Indian Geotechnical Journal. 2017 Dec 1; 47(4):421-36.

[5] Xia Z, Kiratitanavit W, Facendola P, Thota S, Yu S, Kumar J, Mosurkal R, Nagarajan R. Fire resistant polyphenols based on chemical modification of bio-derived tannic acid. Polymer Degradation and Stability. 2018 Jul 1; 153:227-43.Arunkumar, N., Venkataraman, V., Thivyashree, Lavanya. A moving window approximate entropy based neural network for detecting the onset of epileptic seizures [J]. International Journal of Applied Engineering Research, 2013, 8 (15):1841-1847. 